\title{
Using GPS, accelerometry and heart rate to predict outdoor graded walking energy
}

expenditure

P.-Y. de Müllenheim ${ }^{a}$, S. Chaudru ${ }^{b}$, M. Emily ${ }^{c}$, M. Gernigon ${ }^{d, e}$, G. Mahé ${ }^{b, f}$, S. Bickert ${ }^{d}, J$. Prioux $^{a, g}$, B. Noury-Desvaux ${ }^{\mathrm{d}, e, \mathrm{~h}}$, and A. Le Faucheur ${ }^{\mathrm{a}, \mathrm{b}, \mathrm{g}}$

${ }^{a}$ Movement, Sport and Health Laboratory (EA 1274), Faculty of Sport Sciences Rennes 2 University

Avenue Charles Tillon

CS 24414

Rennes, F-35044, France

bINSERM CIC 1414, Clinical Investigation Center

University Hospital of Rennes

Pavillon Clemenceau

2 Rue Henri Le Guilloux

Rennes, F-35033, France

IRMAR, CNRS UMR 6625

Agrocampus Ouest

65 Rue de Saint-Brieuc

CS 84215

Rennes, F-35042, France

'Laboratory for Vascular Investigations and Sports Medicine

University Hospital of Angers

4 Rue Larrey

Angers, F-49933, France

eLaboratory of Physiology, INSERM UMR 1083, CNRS UMR 6214, Medical School University of Angers

Rue Haute de Reculée

Angers, F-49045, France

fHeart-Vessels Imaging Team

Université de Rennes 1, University Hospital of Rennes

Pavillon Clemenceau

2 Rue Henri Le Guilloux

Rennes, F-35033, France

9Department of Sport sciences and physical education

Ecole normale supérieure de Rennes

Campus de Ker Lann 
Bruz, F-35170, France

hInstitute of Physical Education and Sports Sciences (IFEPSA)

Université Catholique de l'Ouest (UCO)

49 Rue des Perrins

BP 40071

Les Ponts-de-Cé, F-49136, France

\section{Corresponding author:}

\section{Alexis LE FAUCHEUR}

Department of Sport sciences and physical education. ENS Rennes. Campus de Ker Lann. Bruz, F-35170, France. Tel: +33 (0)2 9905 9419. Fax: +33 (0)2 99059329 . E-mail: alexis.lefaucheur@ens-rennes.fr

\section{Abstract}

Objectives: To determine the best method and combination of methods among global positioning system (GPS), accelerometry, and heart rate (HR) for estimating energy expenditure (EE) during level and graded outdoor walking.

Design: Thirty adults completed 6-min outdoor walks at speeds of $2.0,3.5$, and $5.0 \mathrm{~km} \cdot \mathrm{h}^{-1}$ during three randomized outdoor walking sessions: one level walking session and two graded (uphill and downhill) walking sessions on a $3.4 \%$ and a $10.4 \%$ grade. EE was measured using a portable metabolic system $\left(K 4 b^{2}\right)$. Participants wore a GlobalSat® DG100 GPS receiver, an ActiGraph ${ }^{\mathrm{TM}}$ wGT3X+ accelerometer, and a Polar® HR monitor. Linear mixed models (LMMs) were tested for EE predictions based on GPS speed and grade, accelerometer counts or HR-related parameters (alone and combined). Root-mean-square error (RMSE) was used to determine the accuracy of the models. Published speed/grade-, count-, and HR-based equations were also cross-validated.

Results: According to the LMMs, GPS was as accurate as accelerometry (RMSE $=0.89$ $\left.0.90 \mathrm{kcal} \cdot \mathrm{min}^{-1}\right)$ and more accurate than $\mathrm{HR}\left(\mathrm{RMSE}=1.20 \mathrm{kcal} \cdot \mathrm{min}^{-1}\right)$ for estimating $\mathrm{EE}$ during level walking; GPS was the most accurate method for estimating EE during both level and uphill $\left(\mathrm{RMSE}=1.34 \mathrm{kcal} \cdot \mathrm{min}^{-1}\right) /$ downhill $\left(\mathrm{RMSE}=0.84 \mathrm{kcal} \cdot \mathrm{min}^{-1}\right)$ walking; combining 
methods did not increase the accuracy reached using GPS (or accelerometry for level walking). The cross-validation results were in accordance with the LMMs, except for downhill walking.

Conclusions: Our study provides useful information regarding the best method(s) for estimating EE with appropriate equations during level and graded outdoor walking.

Keywords: global positioning system, accelerometer, exercise, energy metabolism, public health, methods.

\section{Introduction}

Walking, the most popular physical activity (PA), has a substantial impact on public health and is a leading therapeutic modality. ${ }^{1}$ An accurate assessment of walking-related energy expenditure (EE) is crucial to determine its overall biological impact on the human body ${ }^{2}$ and appropriately study its effects on health. From this perspective, accurately estimating walking EE in outdoor settings is of primary interest. Indeed, it has been shown that i) walkers prefer neighborhood streets and parks for walking; ${ }^{3}$ ii) accumulating walking trips at a sufficient intensity ( $\geq 3$ metabolic equivalents of task (METs)) and of sufficient duration ( $\geq 10 \mathrm{~min})$ to meet PA recommendations ${ }^{4}$ may be easier outdoors; and iii) outdoor walking sessions are of interest in clinical populations for both walking capacity assessment and rehabilitation purposes. $^{5}$

Because the direct measurement of $E E$ in real-life settings is challenging, wearable devices have been validated and used for years in field-based research to track walking EE. ${ }^{6}$ Historically, wearable devices relied on a single objective method for estimating EE. Accelerometry or heart rate $(\mathrm{HR})$ monitoring have been among the most frequently used objective methods. ${ }^{6}$ However, accelerometry has several well-known drawbacks, including the inability to accurately assess EE during graded walking. ${ }^{7} \mathrm{HR}$ monitoring is of particular interest when measuring walking EE because the method relies on the measurement of the physiological response of a given individual. ${ }^{8}$ Unfortunately, a number of factors are 
responsible for the biological variability of $\mathrm{HR}$, which lowers the accuracy of this method when the intensity of PA (including walking) is low. ${ }^{8}$ Interestingly, we recently showed that the use of speed and grade data obtained using a low-cost global positioning system (GPS) receiver allowed highly accurate estimations of EE during level and uphill outdoor walking compared to the use of actual speed and grade. ${ }^{9}$ Although that study supported the feasibility of using GPS alone for estimating EE during level and graded outdoor walking, it is unknown how GPS compares with classical methods, such as accelerometry or HR monitoring, for this purpose.

Beyond determining the best single method for estimating outdoor walking $E E$, it would be helpful to identify the best combination of methods for this purpose. Technological development has allowed the measurement of PA and EE by various sensors embedded into either several small devices (e.g., an accelerometer and an HR monitor ${ }^{10}{ }^{11}$ ) or a single device, such as the SenseWear Mini Armand $^{12}$ or the Actiheart ${ }^{13}$. Moreover, ubiquitous consumer technologies, such as smartphones and watches, are now likely to embed several sensors (including GPS chipsets, accelerometers, and HR sensors), which has opened new perspectives in the field of mobile health. ${ }^{14}$

Several studies have investigated whether combining accelerometry with other methods could close the gap between actual and estimated EE values during graded walking classically observed when using accelerometry alone. ${ }^{12,15,16}$ Some of these studies have assessed the benefits of combining accelerometry and measurements of altitude change (using barometry ${ }^{15,16}$ or GPS $^{15}$ ) to improve the accuracy of EE estimates during outdoor uphill and downhill walking. The value of combining accelerometry and HR monitoring for estimating walking EE has also been assessed, but only on motorized treadmills, and studies have produced conflicting results: some authors found no improvement in the accuracy of EE estimates, ${ }^{11}$ while others reported an improvement ${ }^{10,17}$. Of note, accurately estimating $E E$ when subjects are walking uphill or downhill seems to remain a challenge when combining accelerometry and physiological parameters. ${ }^{12,13}$ 
To our knowledge, no study has compared the accuracy of using different combinations of parameters, including GPS speed and grade, accelerometer counts, and HR parameters, for estimating outdoor walking EE. Therefore, we still do not know the optimal combination to be implemented by researchers and users to study outdoor walking EE when several methods are simultaneously available. Furthermore, it seems relevant to assess whether using several combined methods for estimating EE is worthwhile because increasing the number of methods and wearable devices would probably increase the burden perceived by subjects, limiting acceptability and compliance.

Thus, the purposes of the present study were the following: i) to determine which method among GPS, accelerometry, and HR monitoring is the most accurate for estimating EE during level, uphill and downhill outdoor walking; and ii) to determine the extent to which a combination of these methods increases the accuracy of EE estimates.

\section{Methods}

This cross-sectional study was part of the "Acti-GPS" project (NCT01805219), which was approved by the local Institutional Ethics Committee (CPP OUEST II, Angers). A total of 30 healthy participants were recruited for the present study, as recommended for criterionrelated validity studies. ${ }^{18}$ Most of participants (26/30) were recruited from the Department of Sports Sciences and Physical Education of the University of Angers and the University of Rennes. The participants were between 20 and 30 years of age, with equal numbers of men and women. Each participant signed an informed consent form and underwent an inclusion visit, a resting metabolic rate measurement, a peak oxygen uptake $\left(\mathrm{V}^{2 p e a k}\right)$ measurement during an incremental field-running test, and three outdoor walking sessions. The incremental running test and the outdoor walking sessions were separated by at least $24 \mathrm{~h}$. In addition, the three outdoor walking sessions, which corresponded to three different levels of grade $(0.0 \%, 3.4 \%$ and $10.4 \%$ mean grades), were performed on 2 or 3 different days, with at least one and a half hours between two sessions. The sequential order of the outdoor walking sessions for each participant was counterbalanced according to the number of grade 
conditions $(n=3)$. The methods of the Acti-GPS project have been extensively described elsewhere. $^{9}$

During the inclusion visit, the participants provided their medical histories and underwent a physical examination. Weight, height, and body fat percentage using the skin fold method were measured, as previously reported. ${ }^{9}$

The resting metabolic rate was measured early in the morning (between 07:00 and 10:30) in the supine position and in a noise-free and thermoneutral $\left(22 \pm 1^{\circ} \mathrm{C}\right)$ room. Participants were asked to fast for at least $12 \mathrm{~h}$, to refrain from vigorous PA and alcohol for $24 \mathrm{~h}$, and to sleep for $8 \mathrm{~h}$ or more prior to testing. After a 20 -min adaptation period, gas exchange was recorded over a 30-min period. Resting $\mathrm{HR}$ and gas exchange were measured with a portable metabolic system ( $\mathrm{K}_{4} \mathrm{~b}^{2}$, Cosmed® , Rome, Italy). The $\dot{\mathrm{VO}}_{2 \text { peak }}$ value was determined using the $\mathrm{K}_{4} \mathrm{~b}^{2}$ during an outdoor maximal incremental field-running test performed on a 400 m athletic track. ${ }^{9}$

On 2 or 3 other and different days, each participant completed six-minute walking periods at speeds of $2.0,3.5$ and $5.0 \mathrm{~km} \cdot \mathrm{h}^{-1}$ during each of the three outdoor walking sessions $(0.0 \%$, $3.4 \%$ and $10.4 \%$ mean grades for the three sessions, respectively). During the outdoor walking sessions on the $3.4 \%$ and $10.4 \%$ mean grades, each walking speed was tested uphill and downhill (Supplemental Material (SM) \#1). Thus, each participant completed 15 walking periods through the study: 3 on the level; 6 on the $3.4 \%$ mean grade (3 uphill and 3 downhill); and 6 on the $10.4 \%$ mean grade ( 3 uphill and 3 downhill). The sequence of walking speeds within each walking session was randomized for each participant. The actual walking speed was calculated for each walking period by dividing the total distance walked (measured with an odometer) by the time (measured with a chronometer). At each location, the actual grade of the walking courses was measured every $25 \mathrm{~m}$ along the courses by a certified surveyor using a Trimble S6 Total Station (Trimble, Sunnyvale, CA) and a Trimble R8 Global Navigation Satellite System. All the locations were free of motorized vehicles, 
buildings and dense vegetation. For only one of the two hiking trails, there were trees at one extremity of the pathway.

The $\mathrm{K} 4 \mathrm{~b}^{2}$ was calibrated according to the manufacturer's guidelines before each test, and reliability data have been previously published. ${ }^{9}$ During all walking sessions, participants were equipped with a low-cost DG100 GPS receiver (GlobalSat® Inc., Taipei, Taiwan; $\sim$ AUD80) that recorded at a $1-\mathrm{Hz}$ sampling rate, as previously described. ${ }^{9}$ Participants also wore a wGT3X+ set at a 90-Hz sampling rate on the iliac crest of the hip (ActiGraph ${ }^{\mathrm{TM}}$, LLC, Pensacola, FL; AUD295; firmware 2.2.1). The HR was recorded using the K4b² Polar® belt. All devices were initialized approximately $10 \mathrm{~min}$ prior to each walking session. Synchronization between the different data files was ensured by simultaneously performing a time mark on the $\mathrm{K}_{4} \mathrm{~b}^{2}$ unit and reporting the current Coordinated Universal Time (www.timeanddate.com/worldclock). At the end of each walking session, data were downloaded from all devices to a personal computer using the manufacturers' software. For each walking period, the mean values of weather parameters (temperature $\left({ }^{\circ} \mathrm{C}\right.$ ), relative humidity $\left(\mathrm{RH}\right.$ in \%), wind speed $\left(\mathrm{km} \cdot \mathrm{h}^{-1}\right)$, and barometric pressure $\left.(\mathrm{hPa})\right)$ were also recorded using information provided by French weather stations.

For data analysis, all data were exported to Excel ${ }^{\circledR}$ (Redmond, WA, version 2010) spreadsheets. The absolute resting $\dot{\mathrm{VO}}_{2}$ was recorded as the mean $\dot{\mathrm{VO}}_{2}$ value over the 30 min measurement period. The relative resting $\dot{\mathrm{VO}}_{2}$ was obtained by dividing the absolute resting $\dot{\mathrm{V}}_{2}$ by the participant's body mass. The $\dot{\mathrm{V}} \mathrm{O}_{2 \text { peak }}$ was the highest 30 -s average $\mathrm{V}_{2}$ value measured during the maximal incremental field-running test. Participants were required to have a peak respiratory exchange ratio $\geq 1.10$ and a peak $\mathrm{HR} \geq 95 \%$ of the age-predicted maximum $\mathrm{HR}^{19}$ to ensure that the test value was maximal and to validate the obtained $\dot{\mathrm{V}} \mathrm{Z}_{2 \text { peak }}$ value. The absolute gross $\mathrm{V}_{2}$ values obtained from the different walking sessions were averaged over the last three minutes of each walking period to reflect steady-state exercise. Values were adjusted using terrain coefficients for energy cost prediction, ${ }^{20}$ as 
previously described. ${ }^{9}$ EE was calculated as $\mathrm{kcal} \cdot \mathrm{min}^{-1}=\left[15.913 \times \dot{\mathrm{VO}} 2\left(\mathrm{~L} \cdot \mathrm{min}^{-1}\right)+5.207 \times\right.$ $\left.\dot{\mathrm{V} C O}\left(\mathrm{~L} \cdot \mathrm{min}^{-1}\right)\right] / 4.186 .^{21}$

For each walking period, the GPS speed data were averaged over the last three minutes to match the average EE data. ${ }^{9}$ The corresponding mean uncorrected and corrected GPS grades were obtained using raw and corrected altitude data, respectively, according to our previously published procedure. ${ }^{9}$ The raw data recorded by the wGT3X+ accelerometer were accumulated into 1-s epoch data (i.e., into counts $\cdot \mathrm{s}^{-1}$ ) using the normal filter to compute the counts per second on the vertical axis (VA) and the vector magnitude (VM). Counts per second for both VA and VM were averaged over the last three minutes of each walking period. The average over the last three minutes of each walking period was calculated for the following HR-related parameters: i) the raw $\mathrm{HR}$ recorded during the walking periods $\left(\mathrm{HR}_{(\mathrm{raw})}\right.$, in beats per minute $(\mathrm{bpm}))$; ii) the $\mathrm{HR}$ above the resting $\mathrm{HR}$ value $\left(\mathrm{HR}_{(\mathrm{aR})}\right.$, in bpm $=\mathrm{HR}_{(\mathrm{raw})}-$ the average $\mathrm{HR}$ recorded during the resting metabolic rate measurement $\left(\mathrm{HR}_{\text {(rest) }}\right)$; and iii) the $H R$ reserve $\left(\mathrm{HR}_{(\mathrm{res})}\right.$, in \%). These $\mathrm{HR}$ parameters have previously been used alone (SM \#2) or in combination with other parameters (e.g., accelerometer counts ${ }^{10,17}$ ) to estimate EE; however, to our knowledge, they have never been tested and compared for the study of outdoor walking.

The first step of our statistical analysis was to test linear mixed models (LMMs). ${ }^{22}$ In that way, we developed equations to predict EE that used GPS speed and grade (uncorrected or corrected), accelerometer counts (VA or VM), HR-related parameters $\left(H R_{(\text {raw }),} \mathrm{HR}_{(\mathrm{aR})}\right.$, or

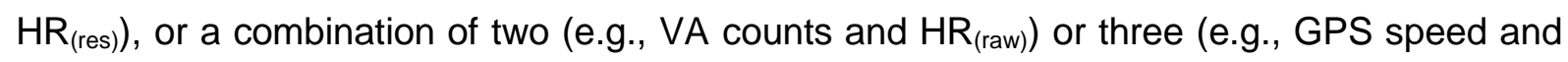
corrected grade, VA counts, and $\left.\mathrm{HR}_{(\mathrm{raw})}\right)$ of these parameters. Separate equations were developed using i) the level walking periods, ii) both the level and uphill walking periods, or iii) both the level and downhill walking periods. We developed separate equations because the relationship between the energy cost of walking and the gradient differs according to the gradient condition (uphill vs. downhill). ${ }^{23}$ The use of both the level and graded walking periods to develop a single equation is in accordance with previous works and the 
components of commonly used equations. ${ }^{4,24}$ Of note, the models that used GPS speed and grade were also compared to the models that used the actual speed and grade. The addition of the following covariates into the models was also tested: mass, gender, body fat percentage, and discomfort index (forward stepwise selection procedure). The discomfort index (thermohygrometric index ${ }^{25}$ ) was calculated as follows: $t-(0.55-0.0055 \cdot R H) \times(t-14.5)$, with air temperature (t) measured in ${ }^{\circ} \mathrm{C}$ and $\mathrm{RH}$ in \%. The coefficient of determination $\left(R^{2}\right)$, standard error of the estimate (SEE) and Akaike information criterion (AIC) were reported for each model. The higher the $R^{2}$ and the lower the SEE, the better the predictive model. The lower the AIC value, the better the quality of the model. The AIC value reflects both the goodness of fit and the "efficiency" (parsimony) of a model for a given number of predictor variables included in the model. An efficient or parsimonious model accomplishes a desired level of prediction with as few predictor variables as possible. The accuracy of the LMMs was tested using the leave-one-out (LOO) procedure ${ }^{26}$ and was reported as the average rootmean-square error (RMSE): the lower the RMSE, the higher the accuracy. Statistical modeling was performed using $R$ software ( $R$ Foundation for Statistical Computing, Vienna, Austria, version 3.2.4).

The second step of our statistical analysis was to cross-validate available published equations for $\mathrm{EE}\left(\mathrm{kcal} \cdot \mathrm{min}^{-1}\right)$ and MET on our own EE $\left(\mathrm{kcal} \cdot \mathrm{min}^{-1}\right)$ and MET data, respectively. These commonly used speed/grade-based, accelerometry-based, and HRbased equations are shown in SM \#2. The cross-validation procedure was performed to quantify any potential "equation effect", that is, a change in the accuracy of the EE estimate according to the prediction equation used. MET values were computed by dividing the absolute gross $\dot{\mathrm{V}}_{2}$ by the body mass plus equipment mass and then by 3.5 . The crossvalidation procedure was performed separately for the level, uphill, and downhill outdoor walking periods, as appropriate. RMSE, bias (95\% confidence interval), limits of agreement and standard deviation of the differences $\left(S_{\text {diff }}\right)$ were computed. The prediction equations were ranked according to the RMSE. 


\section{Results}

\section{Descriptive statistics}

The participant characteristics are shown in Table 1. The medians [interquartile ranges (IQRs) $=25^{\text {th }}-75^{\text {th }}$ percentiles] of the weather parameters during outdoor walking periods were $17.5^{\circ} \mathrm{C}[9.1-20.0], 54.0 \%[34.0-65.4], 11 \mathrm{~km} \cdot \mathrm{h}^{-1}$ [2.0-16.0], and 1019.3 $\mathrm{hPa}$ [999.31023.9] for temperature, $\mathrm{RH}$, wind speed, and barometric pressure, respectively.

All GPS data were correctly recorded, except for the data collected for one participant during the graded outdoor walking session on a 3.4\% mean grade (due to loss of the GPS satellite signal). Regarding the HR data, $10 \%(9 / 90), 7 \%(13 / 180)$, and $13 \%(24 / 180)$ of the values were lost during the level, uphill, and downhill walking periods, respectively, due to a malfunction of the HR sensor. Therefore, $8 \%(22 / 270)$ and $12 \%(33 / 270)$ of the HR values were missing from tests of the level/uphill models and the level/downhill models that used HR data, respectively. All wGT3X+ data were correctly recorded.

The medians $[\mathrm{IQR}]$ for the EE, accelerometer counts, and HR-related parameters measured during the last three minutes of the walking periods are shown in SM \#3. Throughout the range of tested grades, the EE and HR parameters increased exponentially, whereas the accelerometer counts followed a U-shape curve.

\section{Linear mixed models}

All the tested models and their related equations and statistical outcomes are shown in SM \#4. Summaries of the results obtained for the LMMs using the level walking periods, both the level and uphill walking periods, or both the level and downhill walking periods are shown in SM \#5, Table 2, and Table 3, respectively. Except for the models using GPS data only (for which both of the models based on uncorrected and corrected grades are shown), these tables show only the models that reached the lowest RMSE in their respective categories, as defined by both the number of methods combined $(1,2$, or 3$)$ and the type of parameters used (GPS-, accelerometry-, and HR-related parameters). 
Level outdoor walking EE. When each of the methods was used alone without covariates, higher $R^{2}$ values were obtained from the GPS models (SM \#5, $\left.R^{2}=0.75-0.76\right)$, although the error in EE estimation was quite similar when using either GPS speed and grade (RMSE = $\left.0.90-0.91 \mathrm{kcal} \cdot \mathrm{min}^{-1}\right)$ or accelerometer VA counts $\left(\mathrm{RMSE}=0.89 \mathrm{kcal} \cdot \mathrm{min}^{-1}\right.$ ) during level walking. When covariates were added to the single method-based models, the RMSE for accelerometry was slightly lower than for GPS, but the RMSE values for both of these models remained in a narrow range $\left(0.63-0.71 \mathrm{kcal} \cdot \mathrm{min}^{-1}\right)$. When $\mathrm{HR}$ monitoring was used, the RMSE was clearly increased compared to that with GPS or accelerometry, regardless of which HR-related parameter was used (RMSE $\geq 1.20 \mathrm{kcal} \cdot \mathrm{min}^{-1}$ without covariates and $\geq 0.99$ $\mathrm{kcal} \cdot \mathrm{min}^{-1}$ with covariates). Compared with GPS and accelerometry alone, no combination of methods produced a lower RMSE.

Level and graded outdoor walking EE. Table 2 shows the results for the LMMs tested using both the level and uphill outdoor walking periods. First, when the single method-based models ("No combination") were tested without covariates, the lowest RMSE was achieved using the actual speed and grade. The RMSE for the GPS-based models was lower than for the accelerometry-based models (by at least $0.40 \mathrm{kcal} \cdot \mathrm{min}^{-1}$ using corrected GPS grade) and HR-based models (by at least $0.55 \mathrm{kcal} \cdot \mathrm{min}^{-1}$ using corrected GPS grade). The RMSE for the GPS-based models was close to that obtained using the actual speed and grade. A slightly lower RMSE was achieved when using the corrected GPS grade than when using the uncorrected GPS grade (decrease of $0.19 \mathrm{kcal} \cdot \mathrm{min}^{-1}$ without covariates). Second, although combining GPS and HR, or combining the three tested methods, produced slightly higher $R^{2}$ coefficients and lower AIC than GPS alone, no combination of methods reduced the RMSE achieved by the most accurate single GPS-based model. Combining accelerometry and HR produced a lower RMSE than accelerometry or HR alone (RMSE decreased by 0.10 and $0.25 \mathrm{kcal} \cdot \mathrm{min}^{-1}$ for accelerometry and $\mathrm{HR}$, respectively, without covariates).

Table 3 shows the results obtained for the LMMs tested using both the level and downhill outdoor walking periods. Overall, the ranking of the models was similar to that using both the level and uphill outdoor walking periods. Of note, both the RMSE values and the variance 
explained were reduced (e.g., all $R^{2}<0.75$ ) when models were tested using the downhill (Table 3) compared to the uphill walking periods (Table 2).

Effects of covariates. Mass was a significant covariate for most models. Height, gender and the discomfort index were also significant covariates in several models. As shown in SM \#5, Table 2, and Table 3, the addition of significant covariates improved nearly all the models (increased $R^{2}$ and decreased $\mathrm{AIC}$ and RMSE), regardless of i) the walking periods used (level, level/uphill, or level/downhill), ii) the type of parameters used (GPS, accelerometry, or $\mathrm{HR})$, and iii) the number of methods combined $(1,2$, or 3$)$. However, the addition of a covariate did not markedly change the ranking of the models presented above.

\section{Cross-validation of published equations}

Level outdoor walking EE and METs. The results obtained from the cross-validation of other EE and MET prediction equations for level walking are presented in SM \#6. Regarding the EE predictions, the unique GPS-based equation tested (Pandolf et al., 1977; SM \#2) was less accurate than the best accelerometry-based equation (Brooks et al., 2005; SM \#2), with an RMSE of $1.23 \mathrm{kcal} . \mathrm{min}^{-1}$ using uncorrected GPS grade ( $v s$. RMSE $=1.00 \mathrm{kcal} \cdot \mathrm{min}^{-1}$ for the Brooks et al. equation, SM \#2). Regarding MET predictions, the best GPS-based equation (Ludlow and Weyand, 2016; SM \#2) and the best accelerometry-based equation (Leenders et al., 2003; SM \#2) produced similar RMSE values (0.50 and 0.52 MET, respectively). The equation by Rue and Kramer (SM \#2) led to the highest error in the estimation of MET (RMSE = 1.95 MET using corrected GPS grade) among the available GPS-based equations. Previously published HR-based equations were associated with higher RMSEs both for EE (RMSE $\geq 1.80 \mathrm{kcal}^{\mathrm{min}}{ }^{-1}$ ) and METs (RMSE $\geq 1.39 \mathrm{METs}$ ) than for most of the GPS-based or accelerometry-based equations.

Graded outdoor walking EE and METs. Regarding uphill outdoor walking (SM \#7), the GPS-based equations clearly allowed more accurate estimations of EE (RMSE range = 1.60-2.04 $\mathrm{kcal} \cdot \mathrm{min}^{-1}$ ) and MET (RMSE range $=1.18-2.78 \mathrm{METs}$ ) than the accelerometry(RMSE range $=2.34-3.74 \mathrm{kcal} \cdot \mathrm{min}^{-1}$ for EE and 2.12-3.05 for METs) or HR-based equations 
(RMSE range $=1.62-2.88 \mathrm{kcal} \cdot \mathrm{min}^{-1}$ for EE and 1.38-2.53 for METs). Compared to the best accelerometry-based equations, the estimation of EE and METs was more accurate with some of the available HR-based equations (SM \#7). The trend obtained for downhill outdoor walking (SM \#8) was quite different: the highest accuracy for estimating EE (RMSE = 1.31 $\mathrm{kcal} \cdot \mathrm{min}^{-1}$ ) or METs (RMSE $=1.22 \mathrm{METs}$ ) was achieved using the accelerometry-based equations by Brooks et al. (SM \#2), whereas the unique tested GPS-based equation by Rue and $\operatorname{Kramer}(\mathrm{SM} \# 2)$ led to lower accuracy (RMSE $=1.74 \mathrm{kcal} \cdot \mathrm{min}^{-1}$ and $1.57 \mathrm{METS}$, using corrected GPS grade) than the best HR-based equations (RMSE $=1.63 \mathrm{kcal} \cdot \mathrm{min}^{-1}$ for $\mathrm{EE}$ and 1.23 for METs).

\section{Discussion}

The present study aimed to determine the best method and the most valuable combination of methods among GPS, accelerometry, and HR monitoring for estimating EE during outdoor walking. Our analyses led to different conclusions for studies of outdoor walking involving i) level walking only or ii) both level walking and walking under various grade conditions.

\section{What is the best combination of method(s) for estimating level outdoor walking EE?}

The LMMs and cross-validation results showed that GPS is as valuable as accelerometry and more valuable than HR monitoring for estimating EE during level outdoor walking. Interestingly, as shown by the LMMs, no combination of methods increased the accuracy of the EE estimates obtained for GPS or accelerometry alone.

Our finding of equivalence between GPS and accelerometry for estimating level walking EE challenges the use of accelerometry as the preferred method for measuring PA and EE. It has been claimed that from a theoretical point of view, acceleration is more directly reflective of the energy cost than speed. ${ }^{27}$ However, this point of view is not supported by the literature on walking. Indeed, several studies have shown that speed is an important predictor of EE during walking, ${ }^{9}$ and only the study by Brooks et al. ${ }^{21}$ compared the use of speed and acceleration for estimating walking EE in the same sample of subjects. Of note, 
the authors ${ }^{21}$ reported that walking speed was a better predictor of EE (prediction equations with higher $R^{2}$ coefficients) than accelerometer counts (VA) during level outdoor walking.

The differences between the conclusions drawn by Brooks et al. ${ }^{21}$ (superiority of speed on acceleration) and our findings (equivalence between GPS and accelerometry) are likely to be due to the statistical procedures implemented. Brooks et al. ${ }^{21}$ used the $R^{2}$ coefficient and did not cross-validate their equations using the LOO procedure, as we did in the present study. As shown in SM \#5, although higher $R^{2}$ coefficient could be obtained for the GPS-based models vs. the accelerometry-based models, the same errors (RMSE) in EE estimation were finally obtained for both models following the LOO cross-validation.

The error found for HR monitoring seems consistent with reports that showed that HR fails to have a linear relationship with EE at low intensities. ${ }^{8}$ Because of methodological considerations, the walking speeds tested in the present study were in the lower range of human level walking speeds $\left(2-5 \mathrm{~km} \cdot \mathrm{h}^{-1}\right)$. Since cardiorespiratory fitness influences the relationship between $\mathrm{HR}$ and $\mathrm{EE},{ }^{28}$ the $\mathrm{HR}$-EE relationship obtained across the range of walking speeds and grades tested in the present study may have been even more flattened for the fittest subjects. As there were different levels of $\dot{\mathrm{V}}_{2}$ peak among the study's participants, it could be assumed that adding $\dot{\mathrm{VO}}_{2}$ peak as covariate in the HR-based models would have increase their accuracy for predicting walking EE. It was indeed the case, but the accuracy remained inferior to that obtained with the other parameters (data not shown). Importantly, HR is also influenced by factors other than PA intensity. ${ }^{8}$ In this way, the significant effect of the discomfort index on EE observed in the present study underlines a possible detrimental influence of environmental conditions on the prediction of EE when using HR. Of note, contrary to the results of most of laboratory studies, ${ }^{10,17}$ the accuracy of EE estimates during level outdoor walking was not increased by combining HR and accelerometry compared to using accelerometry alone.

The cross-validation results (SM \#6) underline the major impact of the equations used to estimate EE and METs, and they highlight the most suitable equations to estimate outdoor 
level walking EE/METs. Future studies should, however, cross-validate our LMM-derived equations in other samples and compare them to these available published equations.

\section{What is the best combination of method(s) for estimating level and graded outdoor walking EE?}

The LMMs clearly show that GPS offers more accurate estimates of EE for both level and graded (uphill and downhill) outdoor walking than accelerometry or HR monitoring. Further, it appears that no combination of methods provides a better estimation of EE than GPS alone. To the best of our knowledge, this finding is unique and confirms the value of very popular equations ${ }^{4,24}$ developed in studies demonstrating the importance of speed and grade as two powerful predictors of walking EE (see SM \#2 and de Müllenheim et al. ${ }^{9}$ ). The previously mentioned drawbacks of accelerometry during graded walking explain the lower accuracy obtained in the prediction of EE. ${ }^{7}$ However, as suggested by our LMMs, if the GPS method is not available, combining $\mathrm{HR}$ and accelerometer counts could be better than using either method alone (except for both level and downhill walking). This last conclusion is in accordance with previous laboratory studies that combined HR and accelerometry during treadmill walking. ${ }^{13}$

When testing models using GPS data, the use of corrected grades from map projection software was particularly valuable for increasing the accuracy of the prediction of walking EE, particularly for both the level and uphill conditions (Table 2), which is consistent with a previous study. ${ }^{9}$ Although the prediction of walking EE using GPS for both level and downhill conditions was not affected by the use of uncorrected grades (Table 3), based on previous data, ${ }^{29}$ the use of corrected grades, if possible, is preferable.

Again, the cross-validation results (SM \#7, SM \#8) highlight the major impact of the equation used for the prediction of EE and METs. For instance, when using the GPS method to estimate METs during outdoor uphill walking, the ACSM equation ${ }^{4}$ seems to be the most suitable (lowest prediction error). In contrast, the equation provided by Rue and Kramer (SM \#2) was highly inaccurate. The use of this last equation could explain why the GPS method 
did not provide a better EE prediction than accelerometry when considering the downhill walking periods for the cross-validation.

\section{Effects of covariates and statistical considerations}

The finding that mass (mostly) and gender were significant covariates for several models is in accordance with previous studies. ${ }^{21}$ However, to our knowledge, the present study is the first to show that the discomfort index could be a significant covariate in several models when estimating outdoor walking EE. This finding is of importance because outdoor walking can occur under very different atmospheric conditions on a day-to-day basis. However, the discomfort index does not take into account wind speed, and the possibility that high wind speed values might impact walking EE cannot be excluded..$^{30}$ In the present study, adding wind speed as a covariate to the models had no effect on the prediction of walking EE (data not shown).

The $R^{2}$ coefficients obtained for the models tested using the downhill walking periods were lower than those obtained for the models tested using the uphill walking periods. This result may be partly explained by i) the loss of linearity between the EE of walking and the grade when the grade is below $0.0 \%{ }^{23}$ and ii) the lower range of measured (actual) EE values during downhill walking than during uphill walking (SM \#3).

Although the RMSE values were decreased when testing the "level/downhill" models (Table 3) compared to those when testing the "level/uphill" models (Table 2), the median value of the actual EE data used for testing the "level/downhill" models was also decreased. Thus, when the RMSE values were normalized to the median of our EE data, the RMSE values for both conditions were within the same range (data not shown).

\section{Limitations}

Our results regarding the GPS method might not extend to environments that have higher levels of obstruction than those encountered in the present study. However, finding the same experimental conditions as the present study in locations with high levels of obstruction 
would be almost impossible. Environmental obstruction should have only a moderate impact on the final estimation of EE during outdoor walking if the GPS altitude is corrected via map projection software, as proposed here. This issue, however, deserves additional research, particularly in view of free-living and prolonged measurements of walking EE.

The accelerometry-based equations that were cross-validated in the present study were originally validated using count data obtained from an older ActiGraph ${ }^{\mathrm{TM}}$ accelerometer (SM \#2), which may have influenced the EE estimation. Furthermore, equations developed using accelerometer counts remain dependent on the monitor used because activity counts cannot be directly compared across monitors.

Finally, additional studies should be conducted to develop population-specific equations, which was not the aim of the present study. Furthermore, our equations developed herein should be cross-validated in other samples, particularly for level/downhill outdoor walking conditions since prediction equations for EE are lacking.

\section{Conclusions}

This study is the first to determine the best method among GPS, accelerometry and HR for estimating walking EE during level and graded (uphill and downhill) outdoor walking. This work involved testing LMMs using data obtained from participants who walked on both positive and negative grades at different walking speeds. Moreover, for the first time, both speed/grade (GPS)-, accelerometry-, and HR-based published equations were crossvalidated under these conditions.

\section{Practical implications}

- When the estimation of outdoor level walking EE is planned during a session, i) either GPS or accelerometry can be used with similar accuracy; ii) using HR monitoring alone is not recommended; and iii) combining two or three methods adds no value.

- When the estimation of outdoor walking EE is planned during a session of both level walking and various grades, i) GPS should be preferred; ii) the corrected GPS grade 
obtained from map software should be used, if available; iii) combining two or three methods adds no value; and iv) if no GPS receiver is available to the user, combining accelerometry and HR monitoring should be preferred.

- Regardless of the methods used, for either level walking or both level and graded walking, the prediction of EE is greatly influenced by the prediction equation used.

\section{Acknowledgments}

The authors are indebted to Nathan Caron for his help during the experiments. The authors thank Dr. Antoine Bruneau and Pr. Pierre Abraham for their inclusion visits and their useful suggestions regarding the design of the Acti-GPS project. The authors also thank Antoine Gaudry from the Breizh Géo iMmo company for topographical measurements. Marie Gernigon is currently working at the EuroMov, univ. Montpellier, Montpellier, France.

The Acti-GPS project was promoted by the University Hospital of Angers (NCT01805219) and was funded by the Genesia Foundation. Pierre-Yves de Müllenheim received a PhD scholarship from the "École normale supérieure de Cachan-Antenne de Bretagne" (CDSN). Ségolène Chaudru received a PhD scholarship from the Region of Brittany, France (ARED).

"Conflicts of interest: none". 


\section{References}

1. Lee IM, Buchner DM. The importance of walking to public health. Med Sci Sports Exerc 2008; 40(7 Suppl):S512-S518.

2. Thompson D, Peacock O, Western $\mathrm{M}$ et al. Multidimensional physical activity: an opportunity, not a problem. Exerc Sport Sci Rev 2015; 43(2):67-74.

3. Eyler AA, Brownson RC, Bacak SJ et al. The epidemiology of walking for physical activity in the United States. Med Sci Sports Exerc 2003; 35(9):1529-1536.

4. American College of Sports Medicine. ACSM's Guidelines for Exercise Testing and Prescription, 9th ed ed, Philadelphia, PA, Wolters Kluwer/Lippincott Williams \& Wilkins; 2014.

5. Gernigon M, Le Faucheur A, Fradin D et al. Global positioning system use in the community to evaluate improvements in walking after revascularization: a prospective multicenter study with 6-month follow-up in patients with peripheral arterial disease. Medicine (Baltimore) 2015; 94(18):e838.

6. Chen KY, Janz KF, Zhu W et al. Redefining the roles of sensors in objective physical activity monitoring. Med Sci Sports Exerc 2012; 44(1 Suppl 1):S13-S23.

7. Terrier P, Aminian K, Schutz Y. Can accelerometry accurately predict the energy cost of uphill/downhill walking? Ergonomics 2001; 44(1):48-62.

8. Achten J, Jeukendrup AE. Heart rate monitoring: applications and limitations. Sports Med 2003; 33(7):517-538.

9. de Mullenheim PY, Dumond R, Gernigon M et al. Predicting metabolic rate during level and uphill outdoor walking using a low-cost GPS receiver. J Appl Physiol (1985) $2016 ; 121(2): 577-588$.

10. Fudge BW, Wilson J, Easton $\mathrm{C}$ et al. Estimation of oxygen uptake during fast running using accelerometry and heart rate. Med Sci Sports Exerc 2007; 39(1):192-198.

11. Luke A, Maki KC, Barkey $\mathrm{N}$ et al. Simultaneous monitoring of heart rate and motion to assess energy expenditure. Med Sci Sports Exerc 1997; 29(1):144-148. 
12. Vernillo G, Savoldelli A, Pellegrini B et al. Validity of the SenseWear Armband to assess energy expenditure in graded walking. J Phys Act Health 2015; 12(2):178-183.

13. Brage $\mathrm{S}$, Ekelund $\mathrm{U}$, Brage $\mathrm{N}$ et al. Hierarchy of individual calibration levels for heart rate and accelerometry to measure physical activity. J Appl Physiol (1985) 2007; 103(2):682-692.

14. Chow CK, Ariyarathna N, Islam SM et al. mHealth in Cardiovascular Health Care. Heart Lung Circ 2016; 25(8):802-807.

15. Duncan GE, Lester J, Migotsky $S$ et al. Measuring slope to improve energy expenditure estimates during field-based activities. Appl Physiol Nutr Metab 2013; $38(3): 352-356$.

16. Yamazaki T, Gen-No H, Kamijo $\mathrm{Y}$ et al. A new device to estimate VO2 during incline walking by accelerometry and barometry. Med Sci Sports Exerc 2009; 41(12):22132219.

17. Corder K, Brage S, Wareham NJ et al. Comparison of PAEE from combined and separate heart rate and movement models in children. Med Sci Sports Exerc 2005; 37(10):1761-1767.

18. Mahar MT, Rowe DA. Construct validity in physical activity research, Chapter 3, in Physical Activity Assessments for Health-Related Research, Welk GJ, ed. Champaign, IL, Human Kinetics, 2002.

19. Tanaka H, Monahan KD, Seals DR. Age-predicted maximal heart rate revisited. J Am Coll Cardiol 2001; 37(1):153-156.

20. Soule RG, Goldman RF. Terrain coefficients for energy cost prediction. J Appl Physiol 1972; 32(5):706-708.

21. Brooks AG, Gunn SM, Withers RT et al. Predicting walking METs and energy expenditure from speed or accelerometry. Med Sci Sports Exerc 2005; 37(7):12161223.

22. Welk GJ. Principles of design and analyses for the calibration of accelerometry-based activity monitors. Med Sci Sports Exerc 2005; 37(11 Suppl):S501-S511. 
23. Minetti AE, Moia C, Roi GS et al. Energy cost of walking and running at extreme uphill and downhill slopes. J Appl Physiol (1985) 2002; 93(3):1039-1046.

24. Pandolf KB, Givoni B, Goldman RF. Predicting energy expenditure with loads while standing or walking very slowly. J Appl Physiol Respir Environ Exerc Physiol 1977; $43(4): 577-581$.

25. Unger J. Comparisons of urban and rural bioclimatological conditions in the case of a central-European city. Int J Biometeorol 1999; 43(3):139-144.

26. Staudenmayer J, Zhu W, Catellier DJ. Statistical considerations in the analysis of accelerometry-based activity monitor data. Med Sci Sports Exerc 2012; 44(1 Suppl 1):S61-S67.

27. Chen KY, Bassett DR, Jr. The technology of accelerometry-based activity monitors: current and future. Med Sci Sports Exerc 2005; 37(11 Suppl):S490-500.

28. Strath SJ, Swartz AM, Bassett DR, Jr. et al. Evaluation of heart rate as a method for assessing moderate intensity physical activity. Med Sci Sports Exerc 2000; 32(9 Suppl):S465-470.

29. de Müllenheim PY, Chaudru S, Gernigon M et al. Accuracy of a low-cost global positioning system receiver for estimating grade during outdoor walking. Physiol Meas 2016; 37(10):1741-1756.

30. Pugh LG. The influence of wind resistance in running and walking and the mechanical efficiency of work against horizontal or vertical forces. J Physiol 1971; 213(2):255-276. 


\section{Tables}

Table 1. Participants' characteristics

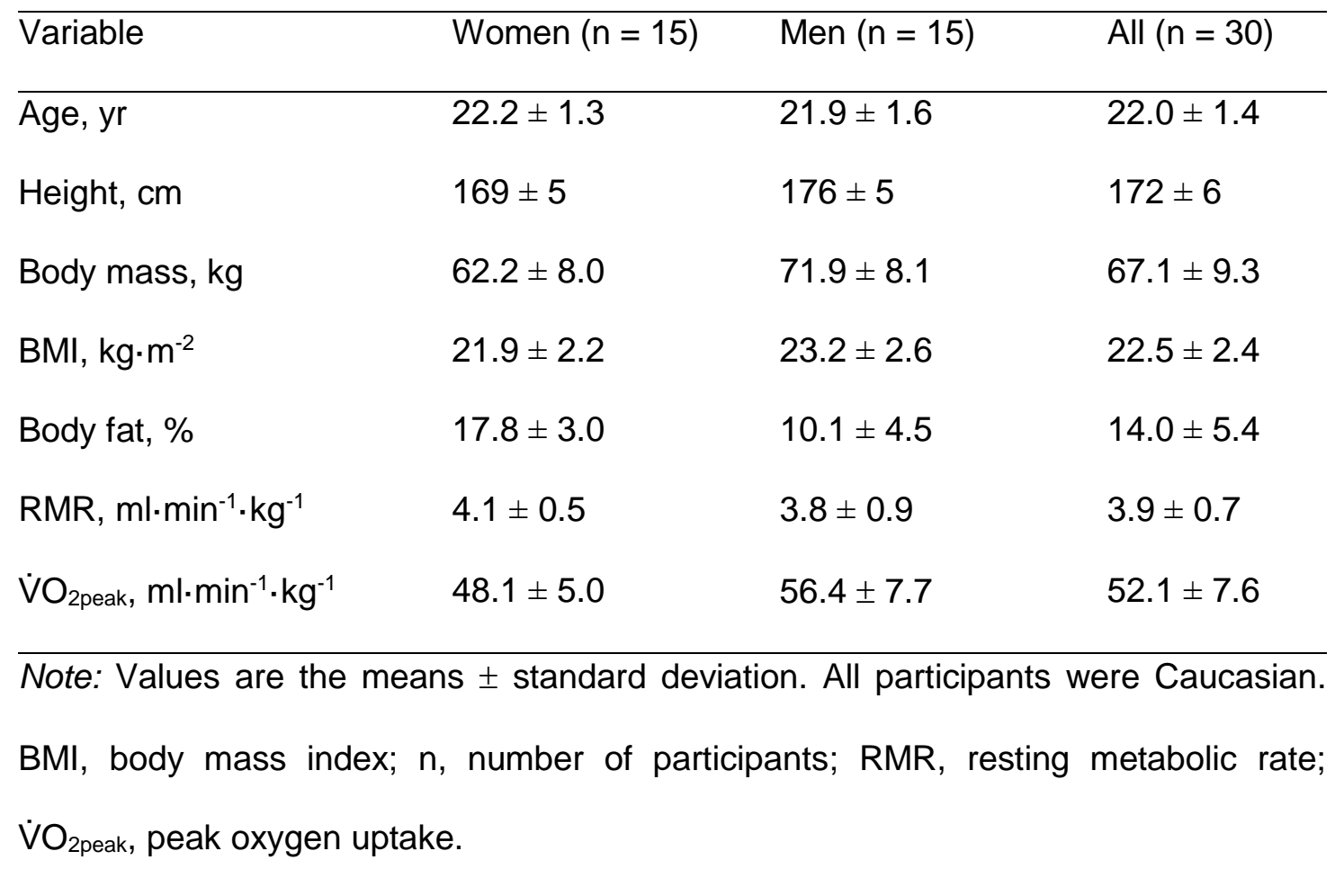


Table 2. Best models for predicting energy expenditure $\left(\mathrm{kcal} \cdot \mathrm{min}^{-1}\right)$ during both level and uphill outdoor walking

\begin{tabular}{|c|c|c|c|c|}
\hline Model & $R^{2}$ & AIC & SEE & RMS \\
\hline \multicolumn{5}{|l|}{ Without covariates } \\
\hline \multicolumn{5}{|l|}{ No combination } \\
\hline Actual $_{\text {(speed \& grade) }}$ & 0.91 & 586.74 & 1.22 & 1.25 \\
\hline GPS $_{\text {(corrected) }}$ & 0.87 & 735.96 & 1.31 & 1.34 \\
\hline $\mathrm{GPS}_{\text {(uncorrected) }}$ & 0.73 & 867.18 & 1.49 & 1.53 \\
\hline $\mathrm{ACC}_{(\mathrm{VM})}$ & 0.58 & 989.67 & 1.71 & 1.74 \\
\hline $\mathrm{HR}_{(\mathrm{aR})}$ & 0.59 & 855.62 & 1.84 & $1.8 \subseteq$ \\
\hline \multicolumn{5}{|l|}{ Combination of 2 methods } \\
\hline $\mathrm{GPS}_{(\text {corrected })}+\mathrm{ACC}_{(\mathrm{VM})}$ & 0.87 & 740.14 & 1.30 & \\
\hline $\mathrm{GPS}_{(\text {corrected })}+\mathrm{HR}_{(\mathrm{aR})}$ & 0.90 & 637.67 & 1.35 & 1.40 \\
\hline $\mathrm{ACC}_{(\mathrm{VM})}+\mathrm{HR}_{(\mathrm{aR})}$ & 0.65 & 814.38 & 1.58 & 1.6 \\
\hline \multicolumn{5}{|l|}{ Combination of 3 methods } \\
\hline $\mathrm{GPS}_{(\text {corrected })}+\mathrm{ACC}_{(\mathrm{VM})}+\mathrm{HR}_{(\mathrm{aR})}$ & 0.90 & 644.49 & 1.34 & 1.40 \\
\hline
\end{tabular}

\section{With covariates}

No combination

\begin{tabular}{|c|c|c|c|c|}
\hline Actual $_{(\text {speed \& grade) }}+D I+M$ & 0.93 & 560.11 & 0.98 & 1.02 \\
\hline $\mathrm{GPS}_{(\text {corrected) }}+M$ & 0.87 & 723.13 & 1.04 & 1.08 \\
\hline $\mathrm{GPS}_{(\text {uncorrected) }}+M$ & 0.80 & 848.00 & 1.24 & 1.27 \\
\hline $\mathrm{ACC}_{(\mathrm{VM})}+M$ & 0.67 & 974.69 & 1.50 & 1.53 \\
\hline $\mathrm{HR}_{(\mathrm{res})}+\mathrm{Gen}$ & 0.67 & 840.28 & 1.52 & 1.58 \\
\hline \multicolumn{5}{|l|}{ Combination of 2 methods } \\
\hline $\mathrm{GPS}_{(\text {corrected })}+\mathrm{ACC}_{(\mathrm{VM})}+M$ & 0.87 & 727.87 & 1.04 & 1.07 \\
\hline $\mathrm{GPS}_{(\text {corrected })}+\mathrm{HR}_{(\mathrm{aR})}+\mathrm{DI}+H t$ & 0.90 & 630.44 & 1.20 & 1.27 \\
\hline $\begin{array}{l}\mathrm{ACC}_{(\mathrm{VM})}+\mathrm{HR}_{(\mathrm{res})}+\text { Gen }+M \\
\text { Combination of } 3 \text { methods }\end{array}$ & 0.76 & 795.08 & 1.25 & 1.32 \\
\hline $\mathrm{GPS}_{(\text {corrected) }}+\mathrm{ACC}_{(\mathrm{VA})}+\mathrm{HR}_{(\mathrm{aR})}+\mathrm{DI}+\mathrm{Ht}$ & 0.89 & 637.15 & 1.18 & 1.26 \\
\hline
\end{tabular}

Notes: The models shown were the most accurate models (i.e., with the lowest RMSE) obtained in their respective categories, which were defined by both the number of parameters combined and the type of parameters used (GPS, ACC, or HR). Covariates appear in italics. The models were ranked according to the RMSE value. Actual (speed \& grade), model using the actual speed and grade; $\mathrm{ACC}_{(\mathrm{VA})}$, model using accelerometry and the vertical axis; $\mathrm{ACC}_{(\mathrm{VM})}$, model using accelerometry and the vector magnitude; AIC, Akaike information criterion; DI, discomfort index; Gen, gender; GPS (corrected), model using both the GPS speed and corrected grade, with the grade calculated using GPS altitude data and corrected with map projection software; GPS (uncorrected), model using both the GPS speed and uncorrected grade; $\mathrm{HR}_{(\mathrm{aR})}$, heart rate above the resting heart rate; $\mathrm{HR}_{\text {(res) }}$, heart rate reserve; $H t$, height; $M$, body mass plus equipment mass; $R^{2}$, R-squared for linear mixed model; RMSE, average root-mean-square error obtained with the leave-one-out methodology; SEE, standard error of estimate. All equations are available in SM \#4. 
Table 3. Best models for predicting energy expenditure $\left(\mathrm{kcal} \cdot \mathrm{min}^{-1}\right)$ during both level and downhill outdoor walking

\begin{tabular}{|c|c|c|c|c|}
\hline Model & $R^{2}$ & AIC & SEE & $R M S$ \\
\hline \multicolumn{5}{|l|}{ Without covariates } \\
\hline \multicolumn{5}{|l|}{ No combination } \\
\hline Actual $_{\text {(speed \& grade) }}$ & 0.68 & 304.87 & 0.81 & 0.84 \\
\hline $\mathrm{GPS}_{\text {(uncorrected) }}$ & 0.67 & 352.31 & 0.82 & 0.84 \\
\hline GPS $_{\text {(corrected) }}$ & 0.67 & 315.78 & 0.82 & 0.85 \\
\hline $\mathrm{ACC}_{(\mathrm{VM})}$ & 0.16 & 686.09 & 1.00 & 1.02 \\
\hline $\mathrm{HR}_{(\mathrm{aR})}$ & 0.29 & 577.83 & 1.07 & 1.09 \\
\hline \multicolumn{5}{|l|}{ Combination of 2 methods } \\
\hline $\mathrm{GPS}_{(\text {corrected })}+\mathrm{ACC}_{(\mathrm{VM})}$ & 0.68 & 324.58 & 0.82 & 0.84 \\
\hline $\mathrm{GPS}_{\text {(corrected) }}+\mathrm{HR}_{(\text {raw })}$ & 0.71 & 292.16 & 0.83 & 0.86 \\
\hline $\mathrm{ACC}_{(\mathrm{VM})}+\mathrm{HR}_{(\mathrm{aR})}$ & 0.29 & 573.93 & 1.01 & 1.04 \\
\hline \multicolumn{5}{|l|}{ Combination of 3 methods } \\
\hline $\mathrm{GPS}_{(\text {corrected) }}+\mathrm{ACC}_{(\mathrm{VM})}+\mathrm{HR}_{(\text {raw })}$ & 0.71 & 300.40 & 0.82 & 0.85 \\
\hline
\end{tabular}

\section{With covariates}

No combination

\begin{tabular}{|c|c|c|c|}
\hline $\mathrm{GPS}_{\text {(uncorrected) }}+D I+M$ & 0.71 & 325.55 & 0.66 \\
\hline Actual $_{(\text {speed } \& \text { grade })}+D I+M$ & 0.74 & 280.39 & 0.66 \\
\hline $\mathrm{GPS}_{(\text {corrected })}+D I+M$ & 0.72 & 281.66 & 0.68 \\
\hline $\mathrm{ACC}_{(\mathrm{VM})}+M$ & 0.38 & 670.97 & 0.86 \\
\hline $\begin{array}{l}\mathrm{HR}_{(\mathrm{aR})}+\mathrm{Ht}+M \\
\text { Combination of } 2 \text { methods }\end{array}$ & 0.49 & 568.56 & 0.87 \\
\hline $\mathrm{GPS}_{\text {(uncorrected) }}+\mathrm{ACC}_{(\mathrm{VA})}+\mathrm{DI}+M$ & 0.71 & 322.07 & 0.67 \\
\hline $\mathrm{GPS}_{\text {(uncorrected) }}+\mathrm{HR}_{(\text {raw })}+\mathrm{DI}+M$ & 0.71 & 307.45 & 0.67 \\
\hline $\begin{array}{l}\mathrm{ACC}_{(\mathrm{VM})}+\mathrm{HR}_{(\mathrm{aR})}+\mathrm{M}+\mathrm{Ht} \\
\text { Jombination of } 3 \text { methods }\end{array}$ & 0.51 & 562.41 & 0.82 \\
\hline $\mathrm{GPS}_{\text {(uncorrected) }}+\mathrm{ACC}_{(\mathrm{VA})}+\mathrm{HR}_{(\mathrm{raw})}+\mathrm{DI}+M$ & 0.71 & 306.16 & 0.68 \\
\hline
\end{tabular}

Note: The models shown were the most accurate models (i.e., with the lowest RMSE) obtained in their respective categories, which were defined by both the number of parameters combined and the type of parameters used (GPS, ACC, or HR). Covariates appear in italics. The models were

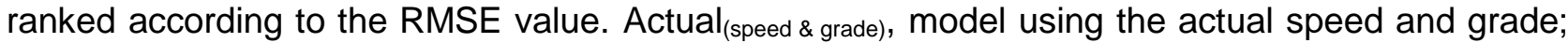
$A C C_{(V A)}$, model using accelerometry and the vertical axis; $A C_{(\mathrm{VM})}$, model using accelerometry and the vector magnitude; AIC, Akaike information criterion; DI, discomfort index; GPS (corrected), model using both the GPS speed and corrected grade, with the grade calculated using GPS altitude data and corrected with map projection software; GPS (uncorrected), model using both the GPS speed and uncorrected grade; $H t$, height; $\mathrm{HR}_{(\text {(raw })}$, raw heart rate; $\mathrm{HR}_{(\mathrm{aR})}$, heart rate above the resting heart rate; $M$, body mass plus equipment mass; $R^{2}$, R-squared for linear mixed model; RMSE, average root-mean-square error obtained with the leave-one-out methodology; SEE, standard error of estimate. All equations are available in SM \#4. 